\title{
A High Efficiency Low Noise RF-to-DC Converter Employing Gm-Boosting Envelope Detector and Offset Canceled Latch Comparator
}

\author{
Thithuy Pham ${ }^{1}$, Dongmin Kim ${ }^{1}$ (D) Seohyeong Jeong ${ }^{1}$, Junghyup Lee ${ }^{2, *}$ and Donggu Im ${ }^{1, *}$ \\ 1 Division of Electronic Engineering, Jeonbuk National University, Jeonju-si 561-756, Korea; \\ thuyphamapc7@gmail.com (T.P.); aksd5736@naver.com (D.K.); eong0229@naver.com (S.J.) \\ 2 Department of Information and Communication Engineering, Daegu Gyeongbuk Institute of Science and \\ Technology (DGIST), Daegu 42988, Korea \\ * Correspondence: jhlee1@dgist.ac.kr (J.L.); dgim@jbnu.ac.kr (D.I.)
}

Citation: Pham, T.; Kim, D.; Jeong, S.; Lee, J.; Im, D. A High Efficiency Low Noise RF-to-DC Converter

Employing Gm-Boosting Envelope Detector and Offset Canceled Latch Comparator. Electronics 2021, 10, 1078 https://doi.org/10.3390/

electronics10091078

Academic Editor: Ahmed Abu-Siada

Received: 17 March 2021

Accepted: 26 April 2021

Published: 2 May 2021

Publisher's Note: MDPI stays neutral with regard to jurisdictional claims in published maps and institutional affiliations.

Copyright: () 2021 by the authors. Licensee MDPI, Basel, Switzerland. This article is an open access article distributed under the terms and conditions of the Creative Commons Attribution (CC BY) license (https:// creativecommons.org/licenses/by/ $4.0 /)$.

\begin{abstract}
This work presents a high efficiency RF-to-DC conversion circuit composed of an LC-CL balun-based $\mathrm{Gm}$-boosting envelope detector, a low noise baseband amplifier, and an offset canceled latch comparator. It was designed to have high sensitivity with low power consumption for wakeup receiver $(\mathrm{WuRx})$ applications. The proposed envelope detector is based on a fully integrated inductively degenerated common-source amplifier with a series gate inductor. The LC-CL balun circuit is merged with the core of the envelope detector by sharing the on-chip gate and source inductors. The proposed technique doubles the transconductance of the input transistor of the envelope detector without any extra power consumption because the gate and source voltage on the input transistor operates in a differential mode. This results in a higher RF-to-DC conversion gain. In order to improve the sensitivity of the wake-up radio, the DC offset of the latch comparator circuit is canceled by controlling the body bias voltage of a pair of differential input transistors through a binary-weighted current source cell. In addition, the hysteresis characteristic is implemented in order to avoid unstable operation by the large noise at the compared signal. The hysteresis window is programmable by changing the channel width of the latch transistor. The low noise baseband amplifier amplifies the output signal of the envelope detector and transfers it into the comparator circuit with low noise. For the $2.4 \mathrm{GHz}$ WuRx, the proposed envelope detector with no external matching components shows the simulated conversion gain of about $16.79 \mathrm{~V} / \mathrm{V}$ when the input power is around the sensitivity of $-60 \mathrm{dBm}$, and this is 1.7 times higher than that of the conventional envelope detector with the same current and load. The proposed RF-to-DC conversion circuit (WuRx) achieves a sensitivity of about $-65.4 \mathrm{dBm}$ based on $45 \%$ to $55 \%$ duty, dissipating a power of $22 \mu \mathrm{W}$ from a $1.2 \mathrm{~V}$ supply voltage.
\end{abstract}

Keywords: baseband amplifier; comparator; conversion gain; envelope detector; LC-CL balun; offset cancellation; programmable hysteresis; RF-to-DC conversion; wake-up receiver

\section{Introduction}

Internet of Things (IoT) is an emerging and fast-growing heterogeneous network that will interconnect a variety of devices (including smartphones, home appliances, sensors, and other network devices), people, data, and processes for a wide range of applications including smart home, building, and cities, agriculture, transportation, and health. Eventually, it allows them to communicate with each other seamlessly. It is expected that the number of connected IoT devices will increase from 27 billion in 2017 to 73 billion in 2025 [1]. One of the most important challenges for a truly innovative IoT solution is extending the lifetime of IoT sensor nodes. Because it is almost impossible to replace the battery periodically in outdoor environments, IoT sensor nodes should be driven by a self-powered energy-harvesting system without a battery by significantly reducing its 
power consumption. The self-powered IoT sensor nodes enable its applications to expand into outdoor environments, and this will give us incalculable economic value.

Various wireless technologies have been proposed for low-power IoT applications, such as IEEE802.15.4 (ZigBee), Bluetooth Low Energy (BLE), IEEE802.15.6 (Medical BodyArea Networks, MBAN), and IEEE802.11a/b/g/n (Wi-Fi) [2-4]. Unfortunately, however, one single wireless technology may not be sufficient to cover both short- and long-range IoT applications. For instance, BLE and ZigBee are competing to provide the IoT with an ultralow-power and low-cost wireless connectivity solution, but their application is limited by the transmission range (within a few hundred meters). Recently, sub-GHz low power wide area network (LPWAN) technologies have been introduced for long range and low-power IoT applications [5,6]. Many LPWAN technologies such as SigFox, long-range wide area network (LoRaWAN), narrowband IoT (NB-IoT), and long-term evolution (LTE)-M have been developed to ensure a long transmission range of more than multiple kilometers with long battery life operation while showing low transmission data rates with small packet data sizes. Consequently, the optimum wireless technology is strongly dependent on IoT applications, and hybrid (short and long range) wireless connectivity solutions optimizing communication range, data rate, and power consumption will be an essential technology in the near future for truly innovative IoT solutions. In addition, because the most power-hungry building block in IoT devices is the wireless communication part, reducing and optimizing the power consumption of the wireless transceiver can make the IoT device intelligent by decreasing the overall power consumption of the complete IoT system and providing opportunities to add many additional functionalities.

It is very important to reduce or eliminate the wasted power in the idle state to decrease the total power consumption of the wireless transceiver. The idle state is when the transceiver is listening to the channel to check for an incoming message [7]. Several techniques such as duty cycling method in [8], asynchronous scheme in [9], and wake-up radio in [10] have been devised to reduce the power in the idle state. Among them, the wakeup radio has been a popular transceiver architecture in recent years for battery-powered IoT applications because of its simple hardware configuration and system application. As shown in Figure 1, the RF to DC converter circuit composed of RF envelope detector (RFED), baseband amplifier, and comparator has been employed as a separate wake-up receiver (WuRx) in order to monitor the communication channel continuously and enable the main radio when it is needed. The most important consideration in implementing a WuRx is low power dissipation while maximizing sensitivity, and as a result, WuRx is typically designed by a simple RFED consisting of Schottky diodes or MOSFETs in the weak inversion region without active filtering or amplification of the input signal [11]. This implies that the sensitivity of WuRx is strongly dependent on the efficiency of the RF to DC conversion and noise performance of RFED. In order to avoid a false wake-up by strong interferers coming from nearby terminals with high transmit power such as mobile phones and WiFi devices, the RFED should have good out-of-band (OOB) rejection characteristic. In addition, it is important to minimize the effect of DC offset of the comparator circuit to avoid sensitivity degradation.

In this paper, a high efficiency RF-to-DC conversion circuit, which is composed of an LC-CL balun-based Gm-boosting RFED, a low noise baseband amplifier, and an offset canceled latch comparator, is proposed for 2.4-GHz WuRx applications. The rest of this paper is organized as follows. In Section 2, we discussed the architecture of the proposed envelope detector. The programmable hysteresis comparator including the proposed DC offset cancellation scheme is analyzed and explained in Section 3. Section 4 discusses the post-layout simulation result and finally, the conclusion is described in Section 5. 


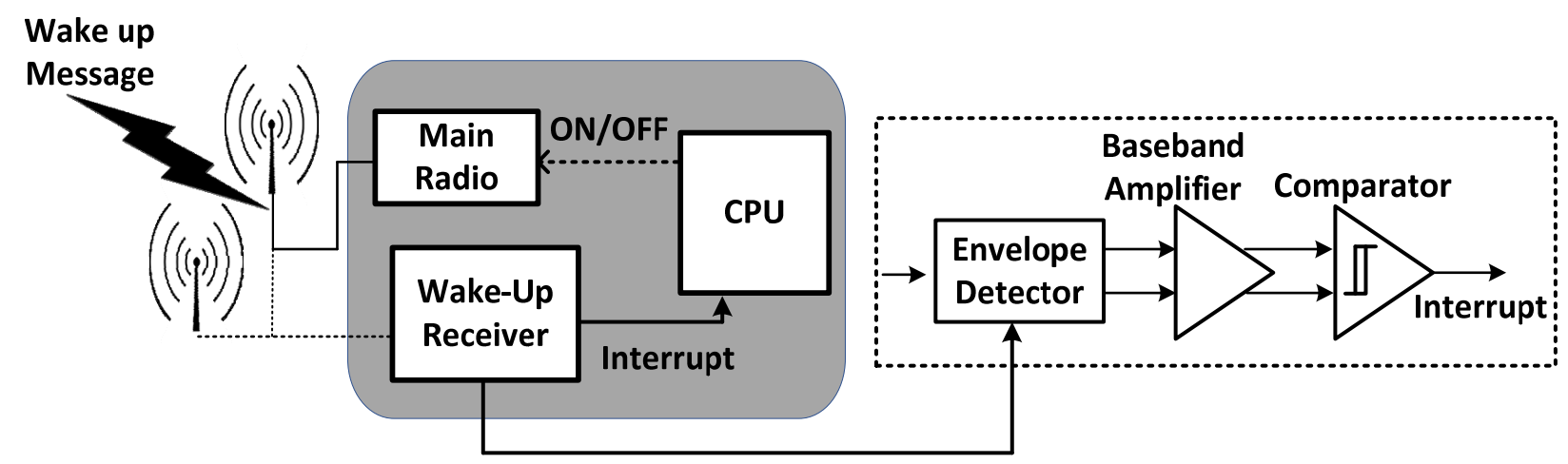

Figure 1. Typical block diagram of a wireless part of IoT sensor node with a separate wake-up receiver.

\section{Design of RF Envelope Detector}

\subsection{Conventional RF Envelope Detector}

Generally, the RF-to-DC conversion exploits the non-linearity feature of devices. There are two types of envelope detector which are passive and active topology. The passive one can operate without a bias current, however, it exhibits various disadvantages such as low input impedance and power loss. The MOSFET with a limited threshold voltage is a key device in an active envelope detection circuit which is commonly used in RF demodulation. Figure 2 shows the schematic of the conventional RFED [12]. It has been built based on the inductively degenerated common-source amplifier with current source load. The envelope of the original signal is extracted by down-converting the incident RF signal $V_{\text {in }}$ to baseband frequencies by using the exponential transfer function of the input transistor $M_{1}$ operating in subthreshold region. The high frequency component is then removed by an $R C\left(R_{1}-C_{1}\right)$ low-pass filter to achieve a pure baseband envelope signal. The most important specifications of an envelope detector are conversion gain, efficiency, and noise performance. The conversion gain is defined as the ratio of output DC voltage (baseband frequency) and input modulated signal (carrier signal). The saturated drain current of the input MOSFET $M_{1}$ biased in the subthreshold region can be expressed as

$$
I_{D} \simeq I_{D 0}\left(\frac{W}{L}\right) \exp \left(\frac{V_{G S}}{n V_{T}}\right)
$$

where $I_{D 0}$ is a current constant independent of gate-to-source voltage, $(W / L)$ is the aspect ratio of the transistor, $n$ is a process-dependent term related to depletion region characteristics, and $V_{T}$ is the thermal voltage ( $=k T / q ; 26 \mathrm{mV}$ at room temperature) [13]. When the small sinusoidal wave $V_{a} \cos \left(w_{c} t\right)$ and DC bias voltage $V_{\text {bias }}$ are applied to the gate of the input transistor $M_{1}$, Equation (1) can be modified based on a Taylor series as

$$
\begin{aligned}
I_{D} & \simeq I_{D 0}\left(\frac{W}{L}\right) \exp \left(\frac{V_{b i a s}+V_{a} \cos \left(w_{c} t\right)}{n V_{T}}\right) \simeq I_{B 0} \exp \left(\frac{V_{a} \cos \left(w_{c} t\right)}{n V_{T}}\right) \\
& \simeq I_{B 0}\left[1+\left(\frac{V_{a}}{2 n V_{T}}\right)^{2}+\frac{V_{a}}{n V_{T}} \cos \left(w_{c} t\right)+\left(\frac{V_{a}}{2 n V_{T}}\right)^{2} \cos \left(2 w_{c} t\right)\right],
\end{aligned}
$$

where $I_{B 0}$ is the DC drain current of the input transistor $M_{1}$. This drain current is converted into the output voltage through a current source with $R C\left(R_{1}-C_{1}\right)$ load, and the $R C$ low-pass filter attenuates the high frequency carrier component of the output voltage leaving a signal DC component $I_{B 0}\left(V_{a} / 2 n V_{T}\right)^{2}$. Because the transconductance $\left(g_{m}\right)$ of the MOSFET biased in the subthreshold region is given by $\left(I_{B 0} / n V_{T}\right)$, the output voltage $\left(V_{o}\right)$ and conversion gain $\left(K_{v}\right)$ of the RFED are calculated as

$$
V_{o} \simeq \frac{1}{4} g_{m} R_{\text {out }}\left(\frac{V_{a}^{2}}{n V_{T}}\right) \text { and } K_{v} \simeq \frac{1}{4} g_{m} R_{\text {out }}\left(\frac{V_{a}}{n V_{T}}\right),
$$


where $R_{\text {out }}$ is the output impedance of the RFED. From (3), the conversion gain, which is the effectiveness of peak detection from carrier frequency to baseband frequency, is directly proportional to $g_{m}, R_{o u t}$, and $V_{a}$. The matching network composed of $L_{2}, C_{2}$, and $L_{3}$ is adopted to match the input impedance of the RFED to the source impedance. The passive voltage gain by the matching network should be added to (3) for the complete conversion gain of the RFED.

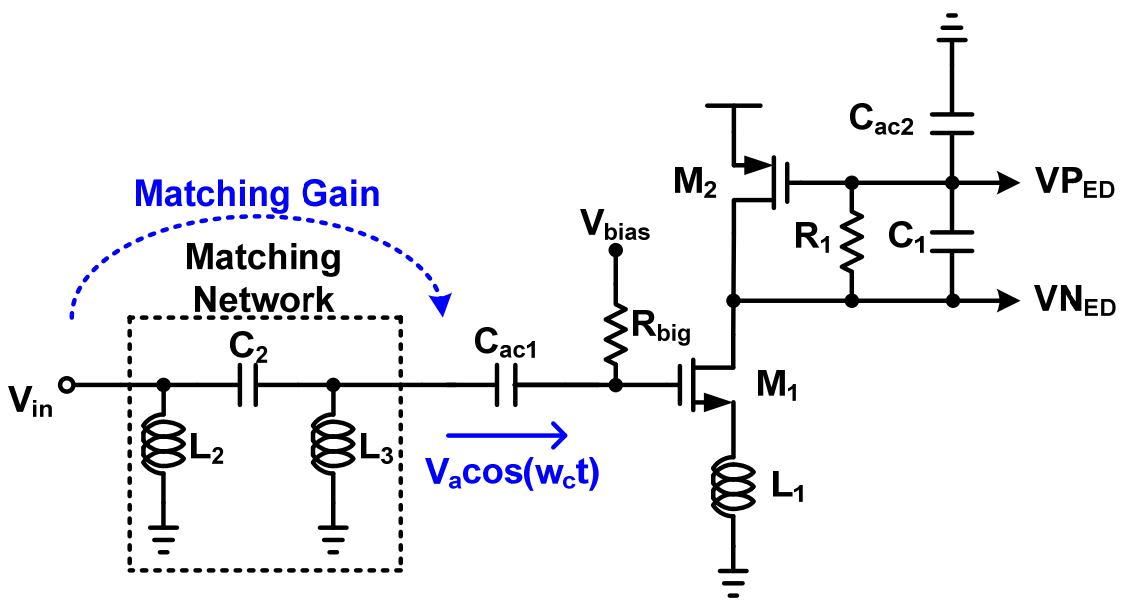

Figure 2. Conventional RF envelope detector.

\subsection{Proposed LC-CL Balun-Based Gm-Boosting RF Envelope Detector}

Figure 3 shows the schematic of the proposed LC-CL balun-based Gm-boosting RFED. Like the conventional RFED of Figure 2, this configuration is originated from an inductively degenerated common source amplifier with a current source. The key part of this detector is the $L C-C L$ balun playing a role as a voltage doubler that converts the single-ended to differential signal across the gate and source of the input transistor $M_{1}$. As a result, the transconductance of input transistor $M_{1}$ is doubled because the gate-source voltage increase twice. This directly increases the conversion gain of the RFED from (3) without any extra power consumption. The input matching network composed of a series capacitor $C_{3}$ and a shunt inductor $L_{3}$ is employed to match the input impedance of the RFED to the source impedance. The $C_{a c 1}$ is a DC blocking capacitor for the input signal. Although this technique requires one additional inductor for the implementation of $L C-C L$ balun, the number of inductors including the matching network is the same as that of the conventional RFED of Figure 2 because of the simpler input matching network.

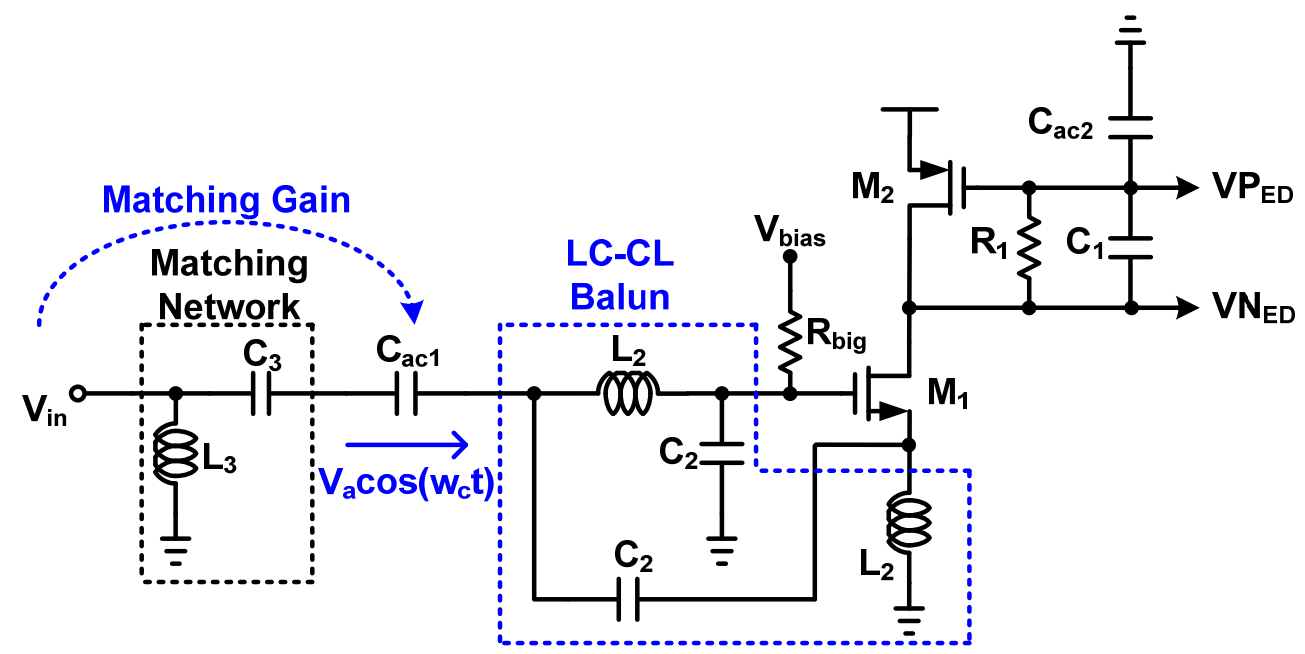

Figure 3. Proposed LC-CL balun-based Gm-boosting RF envelope detector. 
Figure 4 compares the simulated conversion gain of the conventional RFED (Figure 2) and proposed RFED (Figure 3). Input power is scaled in $\mathrm{dB}$ and output voltage is on a logarithmic scale. The summary of device size and component value for both detector circuits is presented in Table 1 . Both detectors are designed to consume an identical current of $10 \mu \mathrm{A}$ from a $1.2 \mathrm{~V}$ power supply, and the value of $R_{1}$ and $C_{1}$ is set to have a cut-off frequency of $50 \mathrm{KHz}$. In addition, all components excepting $C_{a c 2}$ are fully integrated into the single chip for the implementation of highly miniaturized WuRx with low cost. In the simulation, the performance of the RFED is evaluated using an AM signal with a modulation index of 0.75 , a modulation rate of $50 \mathrm{KHz}$, and a carrier frequency of $2.4 \mathrm{GHz}$. As shown in Figure 4, the proposed RFED shows a conversion gain of $16.79 \mathrm{~V} / \mathrm{V}$ which is 1.7 times higher than that of the conventional RFED. The output voltage of the proposed RFED is approximately $2.8 \mathrm{mV}$ for an $-60 \mathrm{dBm}$ input signal.

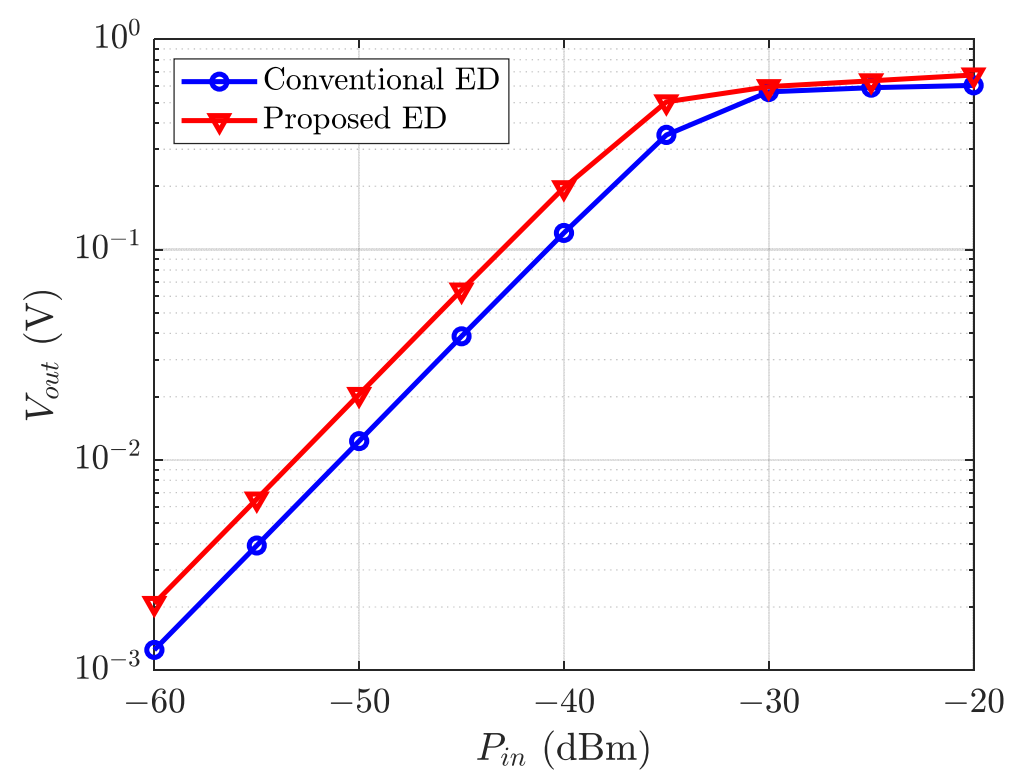

Figure 4. Simulated conversion gain of the conventional and proposed RFED.

Table 1. Component values of the conventional and proposed RFED.

\begin{tabular}{ccccc}
\hline Type & Component & Value & Component & Value \\
\hline Conventional & $\mathrm{M}_{1}$ & $28 \mu \mathrm{m} / 0.24 \mu \mathrm{m}$ & $\mathrm{L}_{3}$ & $6.5 \mathrm{nH}$ \\
RFED & $\mathrm{M}_{2}$ & $90 \mu \mathrm{m} / 0.7 \mu \mathrm{m}$ & $\mathrm{C}_{1}$ & $2 \mathrm{pF}$ \\
& $\mathrm{R}_{1}$ & $1.5 \mathrm{M} \Omega$ & $\mathrm{C}_{2}$ & $0.7 \mathrm{pF}$ \\
& $\mathrm{R}_{\mathrm{big}}$ & $100 \mathrm{k} \Omega$ & $\mathrm{C}_{\mathrm{ac}}$ & $5 \mathrm{pF}$ \\
& $\mathrm{L}_{1}$ & $6.5 \mathrm{nH}$ & $\mathrm{C}_{\mathrm{ac}}$ & $1 \mu \mathrm{F}$ \\
& $\mathrm{L}_{2}$ & $1.3 \mathrm{nH}$ & & \\
\hline Proposed & $\mathrm{M}_{1}$ & $28 \mu \mathrm{m} / 0.24 \mu \mathrm{m}$ & $\mathrm{C}_{1}$ & $2 \mathrm{pF}$ \\
RFED & $\mathrm{M}_{2}$ & $90 \mu \mathrm{m} / 0.7 \mu \mathrm{m}$ & $\mathrm{C}_{2}$ & $0.66 \mathrm{pF}$ \\
& $\mathrm{R}_{1}$ & $1.5 \mathrm{M} \Omega$ & $\mathrm{C}_{3}$ & $12 \mathrm{pF}$ \\
& $\mathrm{R}_{\mathrm{big}}$ & $100 \mathrm{k} \Omega$ & $\mathrm{C}_{\mathrm{ac}}$ & $5 \mathrm{pF}$ \\
& $\mathrm{L}_{2}$ & $6.5 \mathrm{nH}$ & $\mathrm{C}_{\mathrm{ac} 2}$ & $1 \mu \mathrm{F}$ \\
& $\mathrm{L}_{3}$ & $1.2 \mathrm{nH}$ & & \\
\hline
\end{tabular}

\section{Design of Baseband Amplifier}

Figure 5a shows the schematic of the designed fully differential baseband amplifier. It is based on a single-stage operational transconductance amplifier (OTA) biased in the subthreshold region. It amplifies the differential outputs of RFED and reduces the effect of DC offset caused by the next comparator circuit. The $R C\left(R_{1}-C_{1}\right)$ load provides some $\mathrm{OOB}$ rejection characteristic, and the value of $R_{1}$ and $C_{1}$ is chosen for a bandwidth greater than $50 \mathrm{KHz}$. For a low power operation, the tail transistor is biased with as low a current 
as $3 \mu \mathrm{A}$. As shown in the frequency response of Figure $5 \mathrm{~b}$, it shows a maximum voltage gain of $13 \mathrm{~dB}$ at the center frequency of $35.5 \mathrm{KHz}$ and a 3-dB bandwidth of $75 \mathrm{KHz}$ in the simulation.

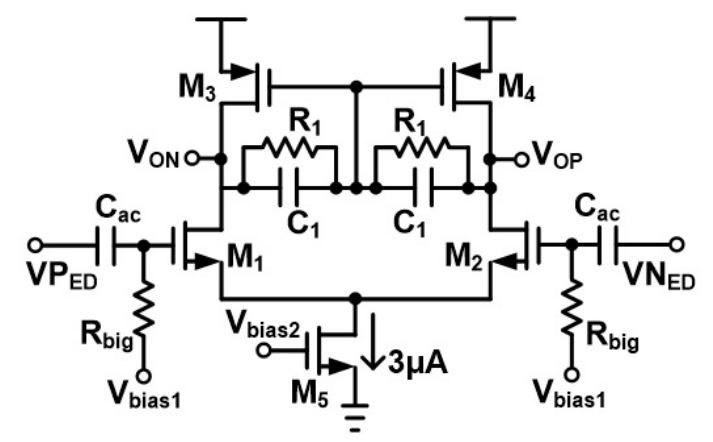

(a)

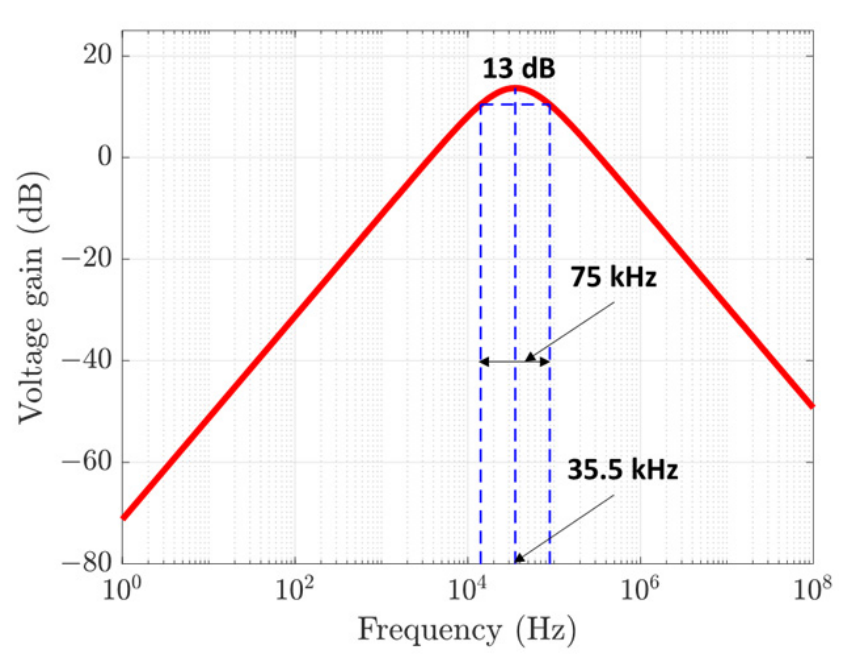

(b)

Figure 5. Schematic and simulation result of designed baseband amplifier: (a) schematic and (b) frequency response.

\section{Design of Comparator}

\subsection{Conventional Hysteresis Comparator}

The hysteresis characteristic is required to avoid unstable operation by the large noise at the compared signal. Figure 6 shows the conventional hysteresis comparator employing internal positive feedback to achieve a hysteresis [14]. The first stage is the PMOS differential pair with diode-connected load transistor $M_{3}\left(M_{6}\right)$ and lath transistor $M_{4}$ $\left(M_{5}\right)$. Intuitively, the amount of hysteresis is determined by the channel width ratio between the diode-connected load transistor and latch transistor because it is directly related to the amount of feedback by latch transistor. The common source stage $M_{7}\left(M_{8}\right)$ amplifies the differential outputs of the first stage, and the differential outputs are converted into the single-ended output through the current mirror $M_{9}$ and $M_{10}$.

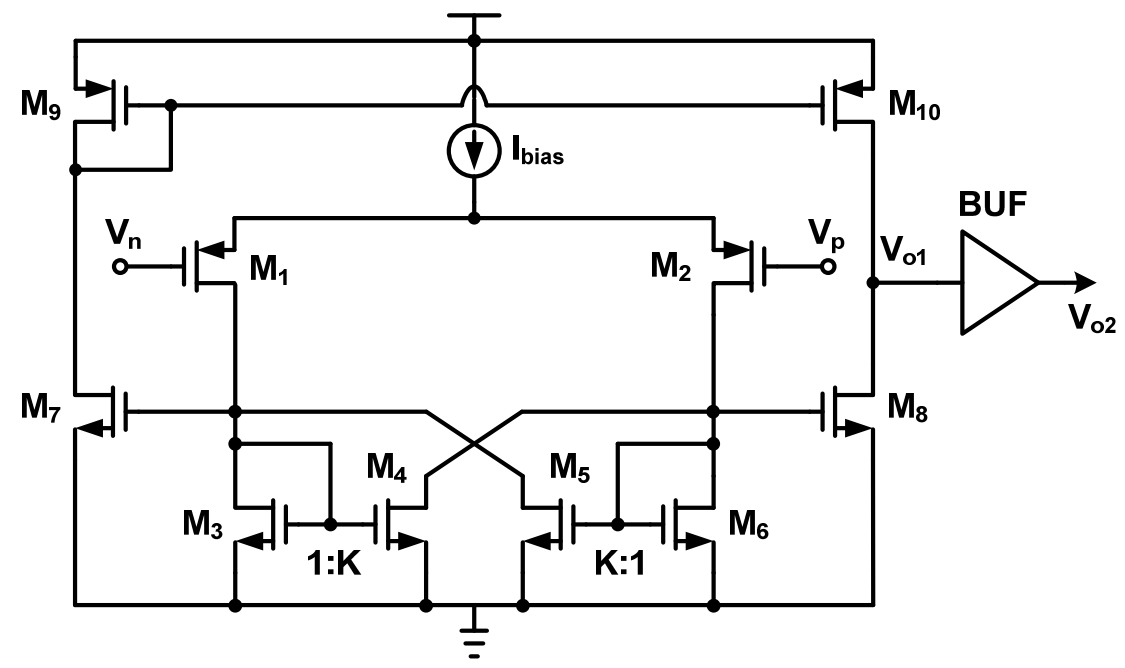

Figure 6. Conventional hysteresis comparator.

When the input signal is higher than the reference voltage by an amount of $V_{\text {hys }}$, the output is at logic high " 1 ". In contrast, the output is at logic low " 0 " when the input 
signal is smaller than the reference voltage by a value of $V_{\text {hys }}$. This relationship can be represented by

$$
V_{o}={ }^{\prime} 1^{\prime} \text { if } V_{p}>V_{n}+V_{h y s} \text { and } V_{o}=^{\prime} 0^{\prime} \text { if } V_{p}<V_{n}-V_{h y s} .
$$

As a result, it is important to calculate the upper trip point $\left(+V_{\text {hys }}\right)$ and lower trip point $\left(-V_{\text {hys }}\right)$ of the comparator circuit. As the differential voltage heads toward the upper trip point, $M_{1}$ turns on. Once it reaches the supply current such that $I_{D 5}=K \cdot I_{D 6}$, where $K$ is the channel width ratio between $M_{3}\left(M_{6}\right)$ and $M_{4}\left(M_{5}\right)$, the comparator trips. The process occurs in a similar way when there is a transition from " 1 " to " 0 ". In summary, the conditions for the upper trip point $\left(+V_{\text {hys }}\right)$ are given by

$$
I_{\text {bias }}=I_{D 1}+I_{D 2}, I_{D 2}=I_{D 6}, I_{D 3}=I_{D 4}=0, \text { and } I_{D 1}=I_{D 5}=K I_{D 6},
$$

where $I_{b i a s}$ is the tail current of the input differential pair. Based on Equation (5), the absolute value of the upper trip point $\left(+V_{h y s}\right)$ and lower trip point $\left(-V_{h y s}\right)$ is derived as

$$
\left|+V_{\text {hys }}\right|=\left|-V_{\text {hys }}\right|=\frac{\sqrt{K}-1}{\sqrt{K+1}} \sqrt{\frac{2 I_{\text {bias }}}{\mu_{p} C_{o x}(W / L)_{1,2}}},
$$

where $\mu_{p}$ is the mobility of input PMOS transistors, $C_{o x}$ is the oxide capacitance, and $(W / L)$ is the ratio between channel width and channel length of input PMOS transistors. The hysteresis window is the difference between these two trip points and can be easily varied by controlling the value of $K$.

\subsection{Proposed Programmable Hysteresis Comparator with Body-Biasing-Based DC Offset Cancellation}

Figure 7 shows the proposed programmable hysteresis comparator with body-biassingbased DC offset cancellation. In order to vary the hysteresis window of the conventional hysteresis comparator of Figure 6, the diode-connected load transistor $M_{3}\left(M_{6}\right)$ and common-source transistor $M_{7}\left(M_{8}\right)$ are constructed with several switched MOSFET branches in parallel, each of which consists of a unit MOSFET in series with a cascode switch. The channel width of unit MOSFET in the switched MOSFET branches is designed to be binary-weighted to vary total channel width linearly adopting the minimum number of branches. The channel length of unit MOSFET in all branches is identical. By digitally turning on or off the cascode switch in each branch, the value of $K$, which is directly related to the feedback factor by latch transistor, can be changed for the programmable hysteresis characteristic.

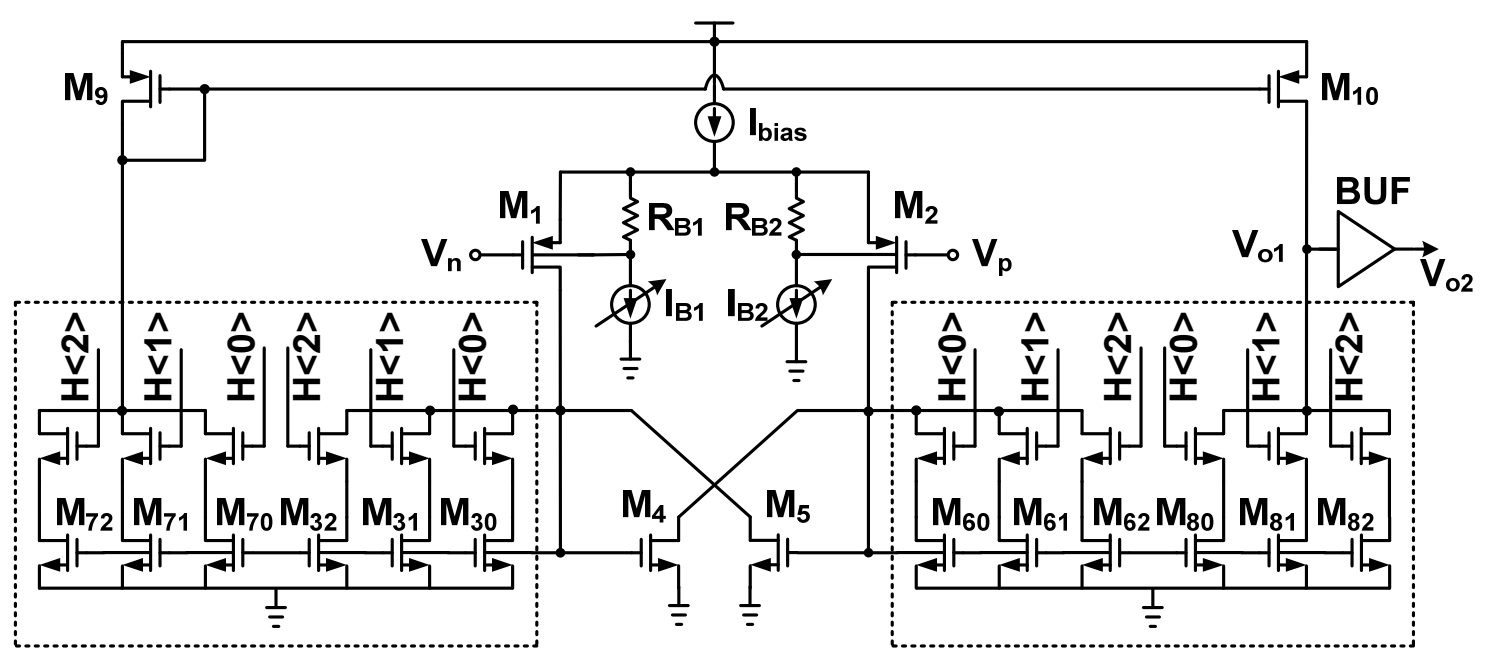

Figure 7. Proposed programmable hysteresis comparator with body-biasing-based DC offset cancellation. 
It is highly required to cancel the DC offset caused by the comparator circuit in order to improve the sensitivity of the wake-up radio. Many offset cancellation techniques, which are based on using pre-amplifiers with input/output offset storage (IOS/OOS) in [15], unbalanced output loads in [16], and auxiliary shunt calibrating transistors at input nodes in [17], have been reported recently. However, the simpler offset cancellation method with extremely low power consumption and small occupied area is highly required for WuRx applications. The work of [18] presented an offset cancellation technique based on a body bias control of the transistor. The offset is sampled and digitalized by the flash analog-to-digital converter (ADC), and the voltage signals generated from the reference digital-to-analog converter (DAC) are injected into the body nodes of the input differential pair to cancel the DC offset. One of the main drawbacks of this technique is that the voltage biasing is quite sensitive to process, voltage, and temperature (PVT) variations. Two voltage signals traveling along a pair of long conductor lines can cause an additional DC offset by some mismatches.

As shown in the proposed DC offset cancellation technique of Figure 7, the current biasing is employed to control the body bias voltage of a pair of differential input transistors. The current generated from the adjustable current source is converted into the voltage near the body node of the input transistor, and this voltage is directly injected into the body node. The hardware complexity of the proposed scheme is very simple, and it is more robust over PVT variations than the conventional voltage biasing-based technique of [18]. Assuming the input offset voltage is modeled as a voltage source, $V_{O S}$, in series with the inverting input node of the comparator and the input voltage $V_{p}$ and $V_{n}$ are the same, $V_{O S}$ can be expressed as

$$
\begin{aligned}
V_{o s} & =-V_{n}-V_{s g 1}+V_{s g 2}+V_{p}=-V_{s g 1}+V_{s g 2}\left(\because V_{n}=V_{p}\right) \\
& \simeq \sqrt{\frac{2 I_{D 2}}{\mu_{p} C_{o x}\left(\frac{W}{L}\right)_{2}}}-\sqrt{\frac{2 I_{D 1}}{\mu_{p} C_{o x}\left(\frac{W}{L}\right)_{1}}}+\left(\left|V_{\text {thp } 2}\right|-\left|V_{t h p 1}\right|\right),
\end{aligned}
$$

where $\left|V_{t h p 1}\right|$ and $\left|V_{\text {thp } 2}\right|$ are the threshold voltage of input transistors $M_{1}$ and $M_{2}$. On the other hand, the threshold voltage of PMOS transistor is given by

$$
\left|V_{t h p}\right| \simeq\left|V_{t h 0, p}\right|+|\gamma|\left(\sqrt{2 \phi_{f}+\left|V_{s b}\right|}-\sqrt{2 \phi_{f}}\right),
$$

where $\left|V_{t h 0, p}\right|$ is the threshold voltage at $V_{s b}=0, \phi_{f}$ is the Fermi voltage, and $\gamma$ is the body-effect coefficient [19]. From (7) and (8), the specific body bias voltages of input transistors can make the exact value of $\left|V_{t h p 2}\right|-\left|V_{t h p}\right|$ to cancel a given DC offset voltage $V_{O S}$ completely.

The programmable hysteresis and offset cancellation characteristics of the proposed comparator are verified through the simulation. Its current consumption is nearly constant for hysteresis characteristics and approximately $2.4 \mu \mathrm{A}$ from a $1.2 \mathrm{~V}$ supply voltage at the initial state without DC offset cancellation. Figure 8a shows the simulated programmable hysteresis window through a digital code. As the 3-bit digital code $H<2: 0>$ decreases from " 111 " to " 001 " in the proposed comparator of Figure 7, the hysteresis window becomes wider $(2.4 \mathrm{mV} \sim 141.6 \mathrm{mV})$. This is well-matched to the predicted result from Equation (6). Figure $8 \mathrm{~b}$ presents the simulated output voltage $\left(V_{o 2}\right)$ of the comparator according to the variation of calibration current $\left(I B_{2}\right)$ when the DC offset voltage $V_{O S}$ ranges from $3 \mathrm{mV}$ to $15 \mathrm{mV}$ with a step size of $2 \mathrm{mV}$. Both of the calibration current $I B_{1}$ and $I B_{2}$ are $125 \mathrm{nA}$ at the initial state, and they are designed to vary from $125 \mathrm{nA}$ to $750 \mathrm{nA}$ with a step size of $125 \mathrm{nA}$ through a digital switch control. It can be seen that the proposed comparator cancels the given DC offset voltage $V_{O S}$ through the adjustment of calibration current $\left(I B_{2}\right)$ and the $I B_{2}$ to make $V_{O 2}$ zero increases when the $V_{O S}$ becomes significant. 


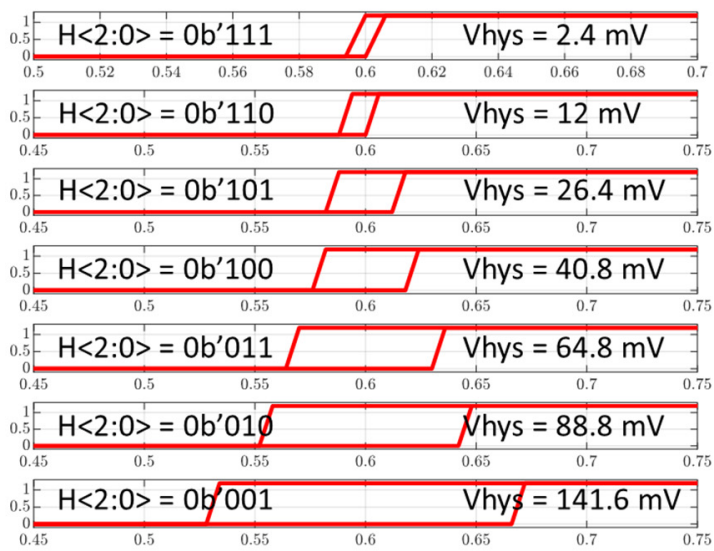

(a)

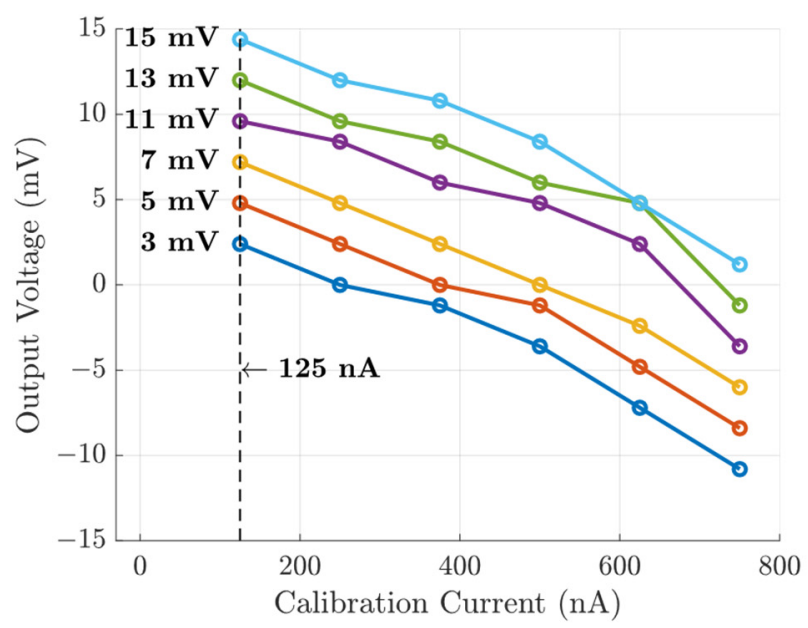

(b)

Figure 8. (a) Simulated programmable hysteresis window through a digital code; (b) simulated output voltage $\left(V_{o 2}\right)$ of the comparator according to the variation of calibration current $\left(I B_{2}\right)$.

\section{Post Layout Simulation Results of Proposed RF-to-DC Conversion Circuit for WuRx}

The proposed high efficiency RF-to-DC conversion circuit for $2.4 \mathrm{GHz}$ WuRx applications was designed and simulated in Cadence/Spectre environment using a 65-nm CMOS process. Figure 9 shows the layout photograph of the designed circuit. It occupies an active area of $500 \times 840 \mu \mathrm{m}^{2}$. All input matching components including inductors are fully integrated into the single chip for the implementation of highly miniaturized WuRx with low cost. The power consumption of the proposed WuRx is $22 \mu \mathrm{W}$ at a $1.2 \mathrm{~V}$ power supply voltage. In order to enhance the accuracy of the simulation, the commercial process design kit (PDK) having high accuracy modeling was used and the post layout simulation removing some errors caused by parasitic resistance and capacitance was proceeded. Because the input matching network is integrated into the chip and all circuit components excepting a by-pass capacitor $C_{a c 2}$ of the proposed RF envelope detector are PDK elements, it is expected that the magnitude of error between the simulation and measurement results is quite small.

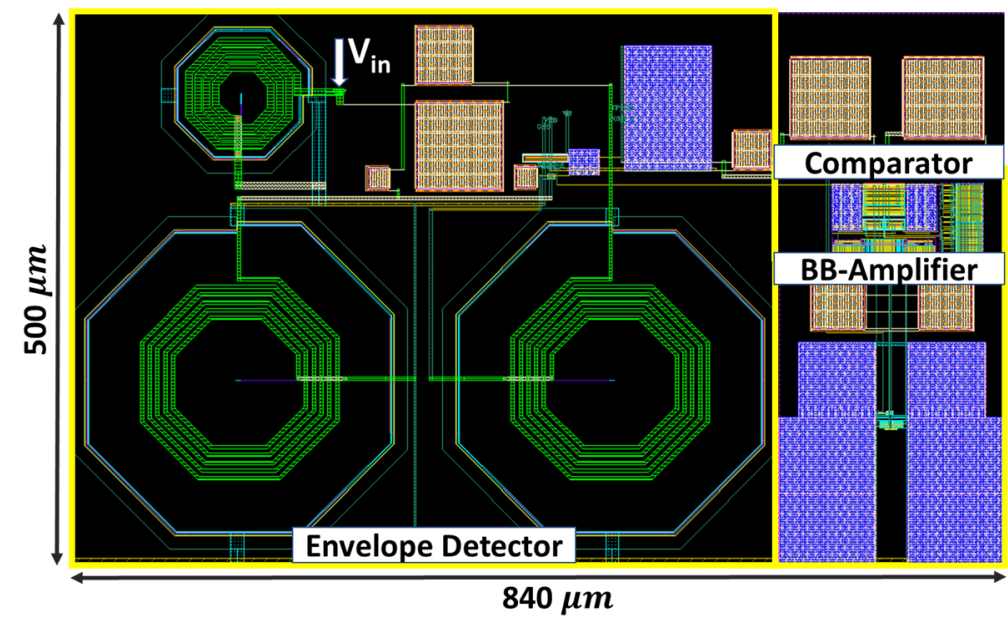

Figure 9. Layout photograph of the designed circuit.

Figure 10 shows the post layout simulated input reflection coefficient $\left(S_{11}\right)$ of the proposed WuRx. The $S_{11}$ of the proposed WuRx is less than $-15 \mathrm{~dB}$ at $2.4 \mathrm{GHz}$. Figure 11 presents the post layout simulated voltage waveforms of the input node of WuRx and output node of RFED, baseband amplifier, and comparator. In the simulation, an AM 
modulated signal with a modulation index of 0.75 , a modulation rate of $50 \mathrm{kHz}$, a carrier frequency of $2.4 \mathrm{GHz}$, and an input power of $-60 \mathrm{dBm}$ is applied to the input of WuRx. When the power of the AM modulated input signal is $-60 \mathrm{dBm}$, the amplitude of the envelope signal at the input node of $\mathrm{WuRx}$ is about $0.8 \mathrm{mV}_{\mathrm{pp}}$. The RFED down-converts it into the baseband frequency and generates the envelope signal with the amplitude of $14 \mathrm{mV}_{\mathrm{pp}}$. The baseband amplifier amplifies the output of RFED with about $13 \mathrm{~dB}$ voltage gain, and the envelope signal with the amplitude of $67 \mathrm{mV}_{\mathrm{pp}}$ is injected into the comparator circuit. The last stage of the comparator generates the final output bit pattern. Based on the requirement of the duty cycle for the common ASK demodulators ranging from $40 \%$ to $60 \%$, the proposed WuRx achieves a sensitivity of $-64.5 \mathrm{dBm}$ for a square-wave bit pattern with $45 \% \sim 55 \%$ duty cycle in the post layout simulation.

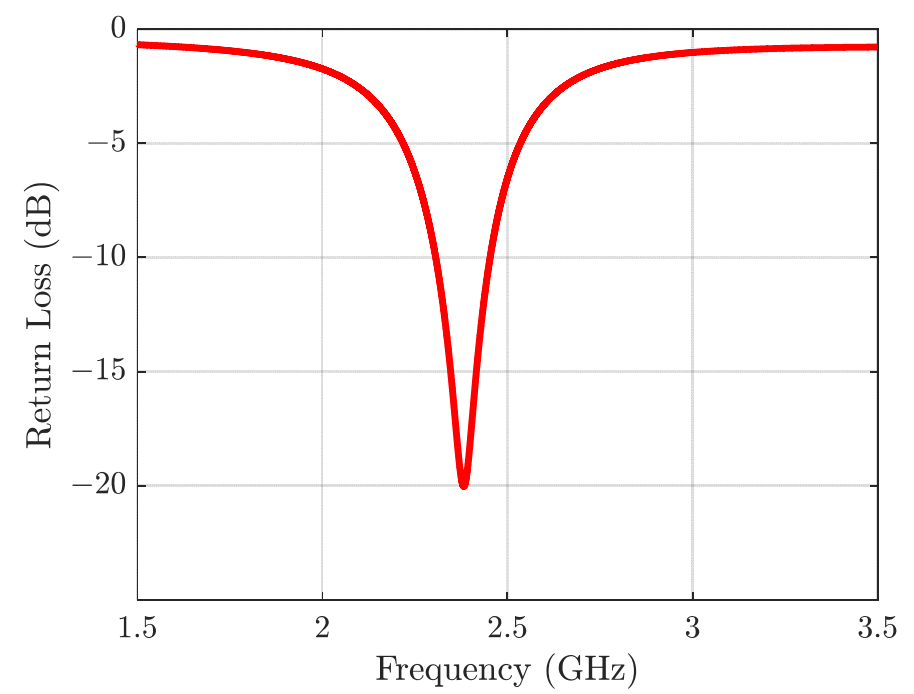

Figure 10. Post layout simulated input reflection coefficient $\left(S_{11}\right)$ of the proposed WuRx.

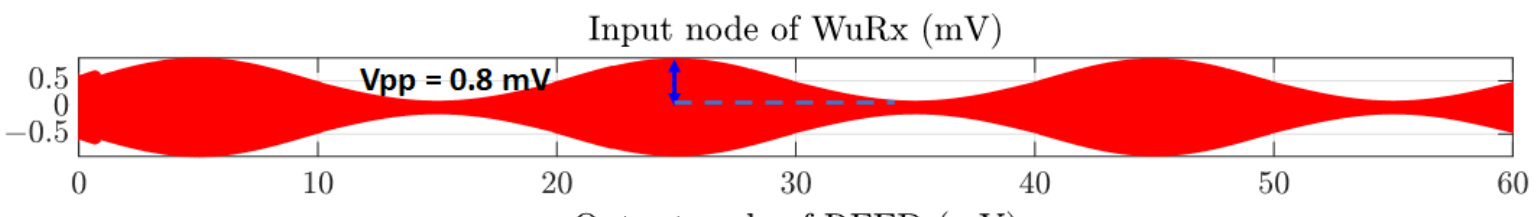

Output node of RFED $(\mathrm{mV})$

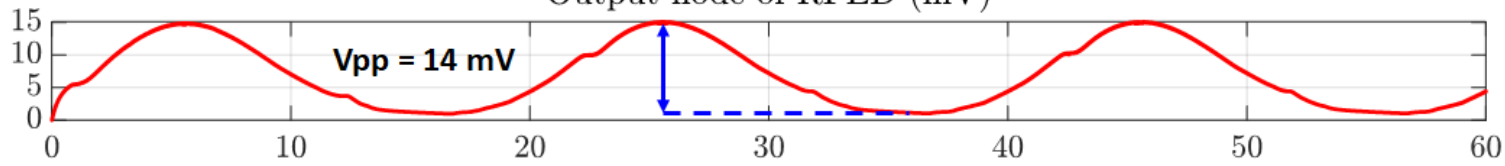

Output node of baseband amplifier $(\mathrm{mV})$

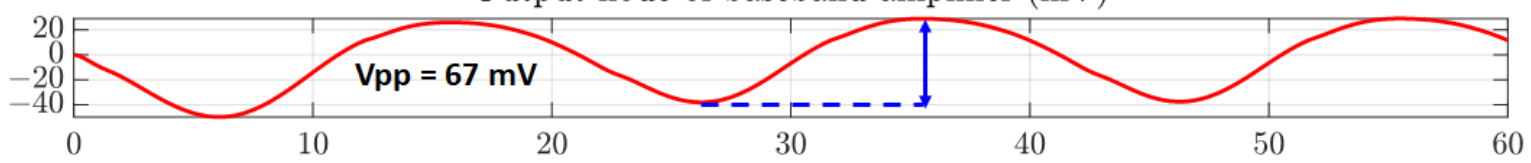

Output node of comparator (V)

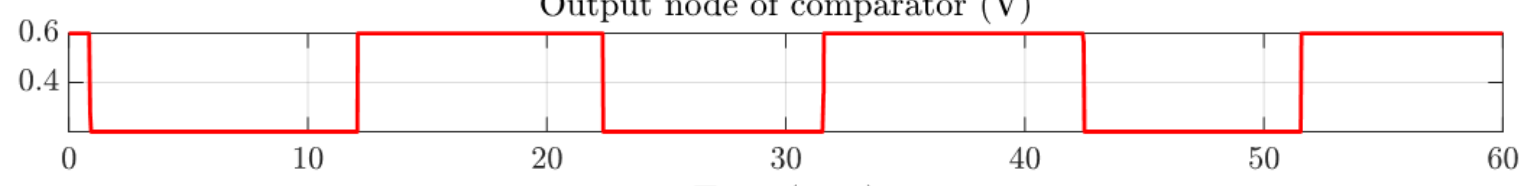

Time $(\mu \mathrm{sec})$

Figure 11. Post layout simulated voltage waveforms of input node of WuRx and output node of RFED, baseband amplifier, and comparator.

In Table 2, the post layout simulation results of the proposed WuRx are summarized and compared to the traditional $2.4 \mathrm{GHz}$ WuRx circuits. One reference work of [20] report- 
ing the simulation results is added in the comparison table. This reference is a touchstone that evaluates the performance of the proposed work because it is designed using the same process technology. Despite of adopting the fully integrated on-chip passive elements for the input matching network, it shows better sensitivity performance while consuming a similar current consumption due to the LC-CL balun-based Gm-boosting technique of RFED and body-biasing-based DC offset cancellation technique of programmable hysteresis comparator. In addition, the proposed WuRx adopting a single-ended RFED can reduce the cost and PCB size by eliminating an external transformer compared to the traditional WuRx circuits with a differential RFED.

Table 2. Performance summary and comparison with the traditional $2.4 \mathrm{GHz}$ WuRx circuits.

\begin{tabular}{|c|c|c|c|c|c|}
\hline Parameters & ${ }^{1}[12]$ & 2 [20] & ${ }^{1}[21]$ & ${ }^{1}[22]$ & 3 This Work \\
\hline $\begin{array}{c}\text { Carrier Frequency } \\
(\mathrm{GHz})\end{array}$ & 2.4 & 2.4 & 2.4 & 2.4 & 2.4 \\
\hline $\begin{array}{c}\text { Modulation Rate } \\
(\mathrm{kHz})\end{array}$ & 100 & 2000 & 100 & 2.5 & 50 \\
\hline $\begin{array}{l}\text { Sensitivity } \\
\quad(\mathrm{dBm})\end{array}$ & -65 & -60 & -50 & -61.5 & -65.4 \\
\hline $\begin{array}{l}\text { Off-chip Matching } \\
\text { Components (?) }\end{array}$ & Yes & No & Yes & ${ }^{4}$ No & No \\
\hline $\begin{array}{l}\text { Differential or } \\
\text { Single-ended }\end{array}$ & Differential & Single-ended & Differential & Single-ended & Single-ended \\
\hline Power Consumption $(\mu \mathrm{W})$ & 10 & 22 & 4.5 & 0.365 & 22 \\
\hline Supply Voltage (V) & 0.5 & 1 & 0.8 & 0.8 & 1.2 \\
\hline CMOS Technology & $180 \mathrm{~nm}$ & $65 \mathrm{~nm}$ & $180 \mathrm{~nm}$ & $65 \mathrm{~nm}$ & $65 \mathrm{~nm}$ \\
\hline
\end{tabular}

${ }^{1}$ Measurement results, ${ }^{2}$ simulation results, ${ }^{3}$ post layout simulation results, ${ }^{4}$ rectifier-antenna co-design.

\section{Conclusions}

The high efficiency RF-to-DC conversion circuit composed of an LC-CL balun-based Gm-boosting RFED, a low noise baseband amplifier, and an offset canceled latch comparator is implemented using a 65-nm CMOS process technology for low power WuRx applications. It provides a new way to increase the conversion gain of RFED and improve the sensitivity by doubling the transconductance of the input transistor without additional power consumption. The programmable hysteresis makes it flexible for the comparator to cover different noise conditions. The offset cancellation scheme, which is implemented without complex circuitry, helps to mitigate the offset due to mismatch error. Taking advantage of technology scaling, the implemented system has become a significant potential solution for many WuRx and wireless communication systems.

Author Contributions: Conceptualization, T.T.P., J.L., and D.I.; investigation, T.T.P., D.K., S.-H.J., J.L., and D.I.; supervision, J.L. and D.I.; writing—original draft, T.T.P. and D.I. All authors have read and agreed to the published version of the manuscript.

Funding: This research was funded by Institute for Information and Communications Technology Promotion (IITP) grant funded by the Korea Government (MSIT) (No. 2018-0-01461) and Basic Science Research Program through the National Research Foundation of Korea (NRF) funded by the Ministry of Education (2018R1A2B6008816).

Acknowledgments: This work was supported by Institute for Information and Communications Technology Promotion (IITP) grant funded by the Korea Government (MSIT) (No. 2018-0-01461) and Basic Science Research Program through the National Research Foundation of Korea (NRF) funded by the Ministry of Education (2018R1A2B6008816). The CAD tool was supported by IDEC.

Conflicts of Interest: The authors declare no conflict of interest. 


\section{References}

1. The Report of IHS Markit, 8 in 2018: The Top Transformative Technologies to Watch This Year. Available online: https: / / www.caba.org/public_research/is-2018-118-8-in-2018-the-top-transformative-technologies-to-watch-this-year (accessed on 3 November 2020).

2. Liu, Y.-H.; Huang, X.; Vidojkovic, M.; Ba, A.; Harpe, P.; Dolmans, G.; De Groot, H. A 1.9 nJ/b 2.4 GHz multistandard (Bluetooth Low Energy/Zigbee/IEEE802.15.6) transceiver for personal/body-area networks. In Proceedings of the 2013 IEEE International Solid-State Circuits Conference-Digest of Technical Papers, San Francisco, CA, USA, 17-21 February 2013 ; pp. $446-447$.

3. Retz, G.; Shanan, H.; Mulvaney, K.; O’Mahony, S.; Chanca, M.; Crowley, P.; Billon, C.; Khan, K.; Quinlan, P. A highly integrated low-power $2.4 \mathrm{GHz}$ transceiver using a direct-conversion diversity receiver in $0.18 \mu \mathrm{m}$ CMOS for IEEE802.15.4 WPAN. In Proceedings of the 2009 IEEE International Solid-State Circuits Conference-Digest of Technical Papers, San Francisco, CA, USA, 8-12 February 2009; pp. 414-415.

4. Kim, S.J.; Kim, D.G.; Oh, S.J.; Lee, D.S.; Pu, Y.G.; Hwang, K.C.; Yang, Y.; Lee, K.Y. A Fully Integrated Bluetooth Low-Energy Transceiver with Integrated Single Pole Double Throw and Power Management Unit for IoT Sensors. Sensors 2019, 19, 2420. [CrossRef] [PubMed]

5. Jovalekic, N.; Drndarevic, V.; Darby, I.; Zennaro, M.; Pietrosemoli, E.; Ricciato, F. LoRa Transceiver with Improved Characteristics. IEEE Wirel. Commun. Lett. 2018, 7, 1058-1061. [CrossRef]

6. Chaudhari, B.S.; Zennaro, M.; Borkar, S. LPWAN Technologies: Emerging Application Characteristics, Requirements, and Design Considerations. Future Internet 2020, 12, 46. [CrossRef]

7. Magno, M.; Benini, L. An ultra low power high sensitivity wake-up radio receiver with addressing capability. In Proceedings of the 2014 IEEE 10th International Conference on Wireless and Mobile Computing, Networking and Communications (WiMob), Larnaca, Cyprus, 8-10 October 2014; pp. 92-99.

8. Jurdak, R.; Baldi, P.; Videira Lopes, C. Adaptive Low Power Listening for Wireless Sensor Networks. IEEE Trans. Mob. Comput. 2007, 6, 988-1004. [CrossRef]

9. Demirkol, I.; Ersoy, C.; Onur, E. Wake-up receivers for wireless sensor networks: Benefits and challenges. IEEE Wirel. Commun. 2009, 16, 88-96. [CrossRef]

10. Jelicic, V.; Magno, M.; Brunelli, D.; Bilas, V.; Benini, L. Benefits of Wake-Up Radio in Energy-Efficient Multimodal Surveillance Wireless Sensor Network. IEEE Sens. J. 2014, 14, 3210-3220. [CrossRef]

11. Choi, J.; Lee, K.; Yun, S.-O.; Lee, S.-G.; Ko, J. An interference-aware 5.8 GHz wake-up radio for ETCS. In Proceedings of the 2012 IEEE International Solid-State Circuits Conference, San Francisco, CA, USA, 19-23 February 2012; pp. 446-448.

12. Cheng, K.-W.; Liu, X.; Je, M. A $2.4 / 5.8 \mathrm{GHz} 10 \mu \mathrm{W}$ wake-up receiver with $-65 /-50 \mathrm{dBm}$ sensitivity using direct active rf detection. In Proceedings of the 2012 IEEE Asian Solid State Circuits Conference (A-SSCC), Kobe, Japan, 12-14 November 2012; pp. 337-340.

13. Van Liempd, B.; Vidojkovic, M.; Lont, M.; Zhou, C.; Harpe, P.; Milosevic, D.; Dolmans, G. A $3 \mu$ W fully-differential RF envelope detector for ultra-low power receivers. In Proceedings of the 2012 IEEE International Symposium on Circuits and Systems (ISCAS), Seoul, Korea, 20-23 May 2012; pp. 1496-1499.

14. Choi, J.; Lee, I.-Y.; Lee, K.; Yun, S.-O.; Kim, J.; Ko, J.; Yoon, G.; Lee, S.-G. A 5.8-GHz DSRC Transceiver With a $10 \mu$ A InterferenceAware Wake-Up Receiver for the Chinese ETCS. IEEE Trans. Microw. Theory Tech. 2014, 62, 3146-3160. [CrossRef]

15. Razavi, B.; Wooley, B.A. Design techniques for high-speed, high-resolution comparators. IEEE J. Solid-State Circuits 1992, 27, 1916-1926. [CrossRef]

16. Van Der Plas, G.; Decoutere, S.; Donnay, S. A 0.16 pJ/Conversion-Step $2.5 \mathrm{~mW} 1.25$ GS/s 4b ADC in a 90 nm Digital CMOS Process. In Proceedings of the 2006 IEEE International Solid State Circuits Conference-Digest of Technical Papers, San Francisco, CA, USA, 6-9 February 2006; pp. 2310-2319.

17. Verbruggen, B.; Craninckx, J.; Kuijk, M.; Wambacq, P.; Van Der Plas, G. A 2.2 mW 5b 1.75GS/s Folding Flash ADC in 90nm Digital CMOS. Proceeding of the 2008 IEEE International Solid-State Circuits Conference-Digest of Technical Papers, San Francisco, CA, USA, 3-7 February 2008; pp. 252-611.

18. Junjie, Y.; Jin, L.; Hoi, L. Bulk Voltage Trimming Offset Calibration for High-Speed Flash ADCs. IEEE Trans. Circuits Syst. II Express Briefs 2010, 57, 110-114. [CrossRef]

19. Mashhadi, S.B.; Nasrollaholosseini, S.H.; Sepehrian, H.; Lotfi, R. An offset cancellation technique for comparators using bodyvoltage trimming. In Proceedings of the 2011 IEEE 9th International New Circuits and Systems Conference, Bordeaux, France, 26-29 June 2011; pp. 273-276.

20. Idres, S.B.; El-Nozahi, M.; Ragai, H.F. A noise cancelling envelope detector for low power wireless sensor applications. In Proceedings of the 2015 IEEE International Symposium on Circuits and Systems (ISCAS), Lisbon, Portugal, 24-27 May 2015; pp. 1302-1305.

21. Chen, S.-E.; Yang, C.-L.; Cheng, K.-W. A $4.5 \mu \mathrm{W} 2.4 \mathrm{GHz}$ wake-up receiver based on complementary current-reuse RF detector. In Proceedings of the 2015 IEEE International Symposium on Circuits and Systems (ISCAS), Lisbon, Portugal, 24-27 May 2015; pp. 1215-1217.

22. Sadagopan, K.R.; Kang, J.; Jain, S.; Ramadass, Y.; Natarajan, A. A 365 nW -61.5 dBm sensitivity, 1.875 cm² 2.4 GHz wake-up receiver with rectifier-antenna co-design for passive gain. In Proceedings of the 2017 IEEE Radio Frequency Integrated Circuits Symposium (RFIC), Honolulu, HI, USA, 4-6 June 2017; pp. 180-183. 